\title{
CLINICAL PROFILE, RISK FACTORS AND INVESTIGATIONS IN PATIENTS WITH RECURRENT ISCHAEMIC STROKE- RESULTS OF A SINGLE CENTRE OBSERVATIONAL STUDY
}

\author{
Ashwinikumar S. Patil1, Ratnakant S. Magdum², Trishala R. Magdum ${ }^{3}$ \\ ${ }_{1}^{1}$ Assistant Professor, Department of Neurology, Seth G. S. Medical College, KEM Hospital, Parel, Mumbai. \\ ${ }^{2}$ Consultant Physician, Mehta Hospital, Sangli, Maharashtra. \\ ${ }^{3}$ Consultant Gynaecologist, Sangli, Maharashtra.
}

\section{ABSTRACT}

\section{BACKGROUND}

The objective of the study was to understand clinical profile, risk factors and usefulness of investigations in patients with recurrent ischaemic stroke.

\section{MATERIALS AND METHODS}

In this observational study, adult patients admitted with recurrent ischaemic stroke were enrolled. Laboratory investigations and imaging studies were performed in these patients. Nature of the previous stroke and risk factors for each patient were recorded.

\section{RESULTS}

Thirty admitted patients (male 76.7\%) were enrolled of which $20(66.7 \%)$ were of 61-80 years of age. 16 (53.4\%) patients were obese or overweight. Hypertension and diabetes were present in $80.0 \%$ and $60 \%$ respectively. Seven $(23.3 \%)$ patients each were smoking and drinking alcohol for more than 10 years respectively. Abnormal cholesterol and HDL were seen in 9 (30\%) each. All the patients were treated with antiplatelets after first ever stroke but only $12(40.0 \%)$ were taking regular treatment whereas only $9(30 \%)$ were continuously taking statins. A total of $27(90 \%)$ were treated by a specialist after first stroke; during followup 21 $(70 \%)$ of the patients showed improvement. In the patients with the study of vasculature $(n=7)$, six $(20 \%)$ had stenosis of arteries and one (3.3\%) had irregular vessel. A total of 16 (53.3\%) of the patients had recurrent stroke in the same territory of blood vessel. Among the patients with same territory involvement $(n=16)$, partial anterior circulation was involved in $8(50 \%)$ patients. Distribution of the patients according to TOAST classification showed large artery atherosclerosis in 12 (40\%) patients. Cardioembolic stroke was present in $2(6.6 \%)$ patients. ECG was normal in 12 (40\%) patients. Echocardiography showed conc. left ventricular hypertrophy in 12 (40\%) patients.

\section{CONCLUSION}

Diabetes mellitus, hypertension, obesity, smoking and alcohol are the common risk factors for recurrent stroke. Most patients had second attack within five years after the first stroke. These findings strongly suggest importance of careful monitoring, clinical examination, followup and treatment adherence in patients with history of stroke.

\section{KEYWORDS}

Investigation, Recurrent Stroke, Risk Factors.

HOW TO CITE THIS ARTICLE: Patil AS, Magdum RS, Magdum TR. Clinical profile, risk factors and investigations in patients with recurrent ischaemic stroke- Results of a single centre observational study. J. Evolution Med. Dent. Sci. 2017;6(34):2808-2812, DOI: $10.14260 /$ Jemds/2017/605

\section{BACKGROUND}

Stroke is a concerning cerebrovascular disease because of its associated mortality and disability. A multinational study estimated the incidence of stroke between 2-2.5/100,000 persons/year. ${ }^{1}$ In India, community surveys have shown a crude prevalence rate of hemiplegia in the range of 200 per 100000 persons. Stroke represents one of the most common and important contributor of all neurological diseases. ${ }^{2}$ Recurrent stroke can cause dementia and potentiate cognitive impairments from other degenerative dementias like Alzheimer's disease. ${ }^{3}$

Financial or Other, Competing Interest: None.

Submission 15-03-2017, Peer Review 14-04-2017,

Acceptance 20-04-2017, Published 27-04-2017.

Corresponding Author:

Dr. Ashwinikumar S. Patil,

Assistant Professor,

Department of Neurology,

Seth G. S. Medical College,

KEM Hospital, Parel, Mumbai.

E-mail: drashwinpatil@gmail.com

DOI: $10.14260 /$ jemds $/ 2017 / 605$
High incidence and cost of management result in economic burden on the country, making stroke and its sequelae an important health care issue for governments and insurance agencies. Overall economic impact makes it imperative to design a cost effective prevention strategy for stroke. ${ }^{3}$ Recurrent stroke is defined as stroke occurring $\geq 24$ hours after the onset of the first stroke, irrespective of vascular territory. 4 About $25 \%-40 \%$ of people who recover from first stroke develop another stroke within five years. ${ }^{5}$ Stroke-related disability increases with each additional episode. Recurrent stroke is the leading cause of stroke mortality particularly high in Eastern Europe and Asia. ${ }^{6}$ The high recurrence rate may partly be attributed to poor compliance to treatment and control of risk factors. ${ }^{7} \mathrm{~A}$ number of risk factors including hypertension, diabetes, hyperlipidaemia, atherosclerotic disease, stress, smoking, and alcohol consumption can lead to stroke. Correction or control of risk factors might reduce the risk of vascular morbidity and mortality in stroke. 5 
Use of effective treatments can reduce the risk of stroke recurrence. ${ }^{8}$ There are limited studies in India regarding risk factors for recurrent stroke.

\section{Objective}

The study was performed to understand clinical profile, risk factors and usefulness of investigations (CT scan and MRI) in patients with recurrent ischaemic stroke.

\section{MATERIALS AND METHODS}

In this observational prospective study, adult patients admitted with recurrent ischaemic stroke diagnosed clinically and patients with previous silent stroke with new onset weakness of limbs were enrolled. Recurrent stroke was defined as occurrence of new onset focal deficit lasting for more than 24 hours and confirmed by imaging studies. Patients below 18 years of age or those with intracranial haemorrhagic stroke were excluded from the study. In patients with speech disturbances, detailed clinical history was obtained from the caregivers. Complete blood count, fasting and postprandial blood sugar, blood urea, serum creatinine, lipid profile, urine analysis, CSF examination, electrocardiogram (ECG), electroencephalogram (EEG), 2DEcho with colour Doppler, computed tomography (CT Scan), magnetic resonance imaging (MRI) of brain, cerebral angiography and carotid Doppler were performed in all patients. Other causes of acute onset focal neurological deficit were excluded by radiological and biochemical tests. Nature of the previous stroke, silent stroke or other problems were determined by clinical and radiological studies. The risk factors for each patient were recorded.

\section{RESULTS}

A total of 30 patients admitted over a period of two years were included in this study. Number and percentage of patients with less than 50 years, 51 - 60 years, 61 - 70 years, 71 - 80 years and more than 81 years were 4 (13.3\%), 4 (13.3\%), 12 (40\%), 8 (26.7\%) and 2 (6.7\%) patients respectively. The age range of patients in this study was $27-$ 84 years. The study included $23(76.7 \%)$ males and 7 (23.3\%) females. The body mass index (BMI) of $14(46.6 \%)$ patients was normal (18.6 - $\left.25 \mathrm{~kg} / \mathrm{m}^{2}\right)$ whereas $9(30 \%)$ patients were obese (BMI $>31 \mathrm{~kg} / \mathrm{m}^{2}$ ). Remaining seven $(23.4 \%)$ patients were overweight (BMI $26-30 \mathrm{~kg} / \mathrm{m}^{2}$ ).

\begin{tabular}{|c|c|c|c|c|}
\hline Family History & Father & Mother & Brother & Wife \\
\cline { 2 - 5 } & $\mathbf{n ~ ( \% )}$ & $\mathbf{n ~ ( \% )}$ & $\mathbf{n ~ ( \% )}$ & $\mathbf{n}(\%)$ \\
\hline Diabetes mellitus & $11(46.7 \%)$ & $5(16.7 \%)$ & $1(3.3 \%)$ & $1(3.3 \%)$ \\
\hline Hypertension & $9(30 \%)$ & 0 & 0 & 0 \\
\hline Rheumatic valvular heart disease (RVHD) & 0 & $1(3.3 \%)$ & 0 & 0 \\
\hline
\end{tabular}

Almost two third of patients were between 61 to 80 years of age group with male predominance $(76.7 \%)$. Large number $(46.6 \%)$ of patients with recurrent stroke had normal BMI.

Eleven $(46.7 \%)$ and five $(16.7 \%)$ patients had history of diabetes in father and mother respectively. History of hypertension in father was present in 9 (30\%) patients (Table 1).

\begin{tabular}{|c|c|}
\hline Risk factors & n (\%) \\
\hline Hypertension & $24(80 \%)$ \\
\hline Diabetes Mellitus & $18(60 \%)$ \\
\hline Stopped treatment & $12(40 \%)$ \\
\hline Alcohol & $11(36.7 \%)$ \\
\hline Obesity & $9(30 \%)$ \\
\hline Smoking & $9(30 \%)$ \\
\hline
\end{tabular}

\begin{tabular}{|c|c|}
\hline Tobacco chewing & $4(13.3 \%)$ \\
\hline Ischemic heart disease (IHD) & $4(13.3 \%)$ \\
\hline RVHD & $1(3.3 \%)$ \\
\hline \multicolumn{2}{|c|}{ Table 2. Risk Factor Profile of the Study Group } \\
\hline
\end{tabular}

The risk factors encountered in the study group were diabetes mellitus, hypertension, obesity, smoking, alcohol, tobacco chewing habits and RVHD. Only one patient with recurrent stroke had no risk factor. Hypertension was most common risk factor $(80.0 \%)$ followed by diabetes mellitus (60\%). Non-compliance to treatment, alcohol consumption, obesity, smoking, tobacco chewing were present in $12(40 \%)$, $11(36.7 \%), 9(30 \%), 9(30 \%)$ and $4(13.3 \%)$ respectively (table 2).

\begin{tabular}{|c|c|c|c|c|c|}
\hline Duration & Diabetes & Hypertension & IHD & Smoking & Alcohol Consumption \\
\hline No & $12(40 \%)$ & $6(20 \%)$ & $26(86.7 \%)$ & $21(70 \%)$ & $19(63.3 \%)$ \\
\hline Less than 5 Years & $1(3.3 \%)$ & $3(10 \%)$ & $1(3.3 \%)$ & 0 & $1(3.3 \%)$ \\
\hline 5 - 10 years & $8(26.7 \%)$ & $11(36.7 \%)$ & $3(10 \%)$ & $2(6.7 \%)$ & $3(10 \%)$ \\
\hline More than 10 years & $9(30 \%)$ & $10(33.3 \%)$ & 0 & $7(23.3 \%)$ & $7(23.3 \%)$ \\
\hline \multicolumn{7}{|c|}{ Table 3. Duration of Common Risk Factors } \\
\hline
\end{tabular}

Twelve (40\%) patients had no diabetes mellitus, nine (30\%) had since more than ten years and eight $(26.7 \%)$ had diabetes since five to ten years. Six (20\%) patients were nonhypertensives. Ten (33.3\%) patients had hypertension since more than ten years whereas $11(36.7 \%)$ and three (10\%) patients had hypertension since 5-10 years and less than five years respectively. Four (13.3\%) patients were suffering from IHD. Two $(6.7 \%)$ patients were smokers since five to ten years whereas seven $(23.3 \%)$ were smoking since more than ten years. Seven $(23.3 \%)$ patients were consuming alcohol since more than ten years.

A total of $18(60 \%)$ of the patients had second stroke within one to five years of the first stroke, whereas six (20\%) each had second stroke after more than five years and within one year. 


\section{Laboratory Investigations}

The number and percentage of patients with abnormal cholesterol, triglyceride, HDL, LDL and VLDL were nine (30\%), six (20\%), nine (30\%), eight (26.7\%) and one (3.3\%) respectively. Out of 30 patients, ten $(33.3 \%)$ had anaemia. Out of ten patients in whom fibrinogen levels were checked, one $(10 \%)$ had high level. Of the eight patients in whom serum homocysteine was evaluated, $37.5 \%$ had high level. Abnormal vitamin B12 level was seen in two (28.5\%) patients out of seven in whom it was measured.

\section{Medication Compliance after first Stroke}

Of all the patients who suffered with first stroke, eight $(26.7 \%)$ stopped treatment since one year whereas two $(6.7 \%)$ patients either stopped the treatment after two to five years or did not take treatment after first ever stroke.

All patients were treated with antiplatelets after first ever stroke; however, only $12(40.0 \%)$ were taking regular treatment. In eight (26.6\%) of the patients, details of treatment was not available whereas ten (33.3\%) patients discontinued the treatment.

Only nine (30\%) patients were continuously taking statins. Ten $(33.3 \%)$ patients discontinued statin treatment whereas in seven $(23.3 \%)$ patients, details of treatment were not available. Four (13.3\%) patients did not take statin at all after first stroke.

Among 24 hypertensive patients, ten (41.6\%) were taking antihypertensive treatment. Details of treatment were not available in six (25\%) patients whereas eight (33.3\%) patients discontinued the treatment.

Among 18 diabetic patients, seven (38.8\%) were taking antidiabetic treatment. Details of treatment were not available in five (27.7\%) patients whereas six (33.3\%) patients discontinued antidiabetic treatment.

\section{Treating Specialty and Followup}

A total of $27(90 \%)$ were treated by a specialist after first stroke whereas only three $(10 \%)$ were treated by a physician. During followup $21(70 \%)$ of the patients showed improvement, two (6.6\%) had irregular followup and seven (23.3\%) never underwent any followup.

\section{Study of Vasculature}

In $23(76.7 \%)$ patients, the vasculature was not studied. In the patients with the study of vasculature $(n=7)$, six $(20 \%)$ had stenosis of arteries and one (3.3\%) had irregular vessel. A total of $16(53.3 \%)$ of the patients had recurrent stroke in the same territory of blood vessel whereas in other 14 $(46.6 \%)$ patients different territory was involved. Among the patients with same territory involvement $(n=16)$, partial anterior circulation infarct (PACI) was involved in eight (50\%) patients. In six (37\%) patients, lacunar infarct (LACI) was involved whereas two $(20 \%)$ patients showed involvement of posterior circulation infarct (POCI) in the first as well as subsequent stroke.

\begin{tabular}{|c|c|}
\hline TOAST Classification & n (\%) \\
\hline Large artery atherosclerosis & $12(40 \%)$ \\
\hline Cardioembolism & $2(6.6 \%)$ \\
\hline Small vessel obstruction & $9(30 \%)$ \\
\hline Stroke of other determined aetiology & $7(23.3 \%)$ \\
\hline
\end{tabular}

Distribution of the patients according to TOAST classification showed large artery atherosclerosis in 12 $(40 \%)$ of the patients whereas nine $(30 \%)$ had small vessel obstruction. Cardioembolism was present in two $(6.6 \%)$ patients.

\section{ECG and ECHO Findings in Stroke Patients}

ECG was normal in $12(40 \%)$ patients. A total of $8(26.6 \%)$ patients showed signs of left ventricular hypertrophy, nine $(30 \%)$ had ischaemic changes whereas one $(3.4 \%)$ patient had ST elevation.

Echocardiography was normal in nine $(30 \%)$ patients. A total of $12(40 \%)$ patients had concentric left ventricular hypertrophy and six (20\%) had degenerative valvular changes. One (3.3\%) patient had shown signs of RVHD.

\section{DISCUSSION}

In this study, we examined the risk factors responsible for recurrent stroke in 30 indoor patients. The age range of patients was 27-84 years, suggesting possibility of stroke in any age group. Advancing age is a known risk factor for stroke. ${ }^{3,9}$ In our study, majority of the patients were between 61 to 80 years of age. In contrary to our findings, Samsa et $\mathrm{al}^{10}$ found that the average age of occurrence of first stroke was 78 years ( \pm 7.5 years). In the recurrent stroke group, the age was 79 years ( \pm 6.9 years). In another study by tho et al, $1114 \%$ of the patients aged below 65 years and $18 \%$ of the subjects above 65 years experienced stroke.

A person's age also influences prognosis of stroke. Higher mortality and lower recovery rates are seen in elderly patients compared to people below 65 years of age. Stroke is an important cause of death and disability in both genders. Ischaemic stroke is more common in men than in women. Most of the patients with our study with recurrent stroke were male $(76.7 \%)$. Women in the reproductive age group (15 - 30 years) have slightly higher risk of stroke due to cerebrovascular events as a complication of pregnancy and puerperium. ${ }^{3}$ Recurrent strokes were slightly more common in females $(53 \%)$ than males $(47 \%)$ in another study by Burn et al. ${ }^{8}$

Overweight men have higher risk of stroke. ${ }^{3}$ The risk from obesity appears to be separate from that with elevated serum cholesterol levels. In our study, over 53\% patients were obese or overweight. Weight reduction is one of the important measures to reduce the risk of ischaemic stroke. ${ }^{12,13}$ Obesity is also a risk factor for other comorbidities such as hypertension, diabetes and dyslipidaemia which in turn can predispose stroke.

None of the patients had history of recurrent stroke or stroke in this study; however, there were risk factors for stroke in the family members. Ischaemic stroke can occur due to inherited disorders of coagulation or genetic disorders predisposing to arterial disease. Familial predisposition to diabetes mellitus may have role in the development of stroke. Familial predisposition to stroke could be contributed by diet, tobacco or access to medical care. ${ }^{3}$ Arterial hypertension is an important and potentially treatable risk factor for stroke.3,14,15 The risk factors encountered in our study were diabetes mellitus, hypertension, obesity, smoking, tobacco and alcohol. Only one patient with recurrent stroke had no risk factor. Elevated blood pressure is associated with higher risk of stroke.16,17 Isolated systolic hypertension is also 
correlated with increased thickness and plaque formation of the internal carotid artery. ${ }^{18}$ In our study, hypertension was most common risk factor (80\%) followed by diabetes mellitus (60\%). Chronic hypertension promotes atherosclerosis. Hypertension is asymptomatic, hence likely to be unrecognised in many patients. Additionally, many patients with arterial hypertension are not adequately treated. ${ }^{19}$ A total of $33.3 \%$ of the patients in this study discontinued the antihypertensive treatment. A history of diabetes is associated with significantly higher risk of stroke. ${ }^{15}$ The severity of and mortality after stroke is also higher among patients with diabetes. In our study, 56.7\% patients had diabetes since more than five years. Diagnosis and management of diabetes improves overall health of the patient. ${ }^{20}$ Hypoglycaemia more commonly mimics clinical features of acute ischaemic stroke than hyperglycaemia. Diabetes is a risk factor for multiple lacunar infarcts. ${ }^{21}$ Most of the diabetes patients in this study either discontinued the treatment or were not taking antidiabetics. The glucose levels in stroke patients should be maintained near to normal, but tight control may increase mortality due to hypoglycaemia, hence is discouraged.

Cigarette smoking is an important risk factor for atherosclerosis, myocardial infarction and ischaemic stroke. ${ }^{3}$ Cessation of tobacco consumption reduces risk. ${ }^{12,22}$ Heavy alcohol consumption may increase the risk of stroke, especially in young persons. ${ }^{23}$ Smoking and alcohol consumption was also reported by many patients in this study. The stroke patients need prophylaxis with the antiplatelet drugs to prevent platelet aggregation of and reduce risk of thromboembolic stroke. Medication noncompliance was common in the study population. Dyslipidaemia and elevated serum homocysteine were common in patients with recurrent stroke. Toole et $\mathrm{al}^{24}$ in an interventional study observed a significant association between serum homocysteine level and stroke.

Overall, our study provides information about risk factors in patients with recurrent stroke. The observation may help clinicians to identify patients with high risk for recurrent stroke and carefully monitor, educate and treat them to prevent complications.

Open label, observational study design, small sample size and absence of control arm are the limitations of this study.

\section{CONCLUSION}

Age, diabetes mellitus, hypertension, obesity/overweight, smoking, alcohol and tobacco chewing habits of longer duration were the common observed risk factors among patients with stroke. Second attack seems to be common within five years after the first attack, hence patients with history of recent stroke should control their risk factors and adhere to the treatment given.

\section{REFERENCES}

[1] Hatano S. Experience from a multicentre stroke register: a preliminary report. Bull WHO 1976;54(5): 541-53.

[2] Dalal M. Cerebrovascular disorders. API textbook of medicine. $7^{\text {th }}$ edn. Association of physician of India 2003;796-8.
[3] Adams HP, Norris JW. Ischemic cerebrovascular disease. CNS Series 2003:1-46.

[4] Coull AJ, Rothwell PM. Underestimation of the early risk of recurrent stroke evidence of the need for a standard definition. Stroke 2004;35(8):1925-9.

[5] Leonberg SC, Elliott FA. Prevention of recurrent stroke. Stroke 1981;12:731-5.

[6] Schoenstadt A. Recurrent stroke. Available from URL: http://stroke.emedtv.com/stroke/recurrentstroke.html.

[7] Kirshner HS. Therapeutic interventions for prevention of recurrent ischemic stroke. Am J Manag Care 2008;14(6 Suppl 2):S212-26.

[8] Burn J, Dennis M, Bamford J, et al. Long-term risk of recurrent stroke after a first-ever stroke. The Oxfordshire community stroke project. Storke 1994;25(2):332-7.

[9] Simons LA, McCallum J, Friedlander Y, et al. Risk factors for ischemic stroke: Dubbo study of the elderly. Stroke 1998;29(7):1341-6.

[10] Samsa GP, Bian J, Lipscomb J, et al. Epidemiology of recurrent cerebral infarction: Medicare claims-based comparison of first and recurrent strokes on 2-year survival and cost. Stroke 1999;30(2):338-49.

[11] Aho K, Harmsen P, Hatano S, et al. Cerebrovascular disease in the community: results of a WHO collaborative study. WHO Bulletin 1980;58(1):113-30.

[12] Linderstrom E, Boysen G, Nyboe J. Influence of total cholesterol, high density lipoprotein cholesterol and triglycerides on risk of cerebrovascular disease: the Copenhagen city heart study. BMJ 1994;309(6946): 11-5.

[13] Lee IM, Paffenbarger RS. Physical activity and stroke incidence: the Harvard Alumni health study. Stroke 1998;29(10):2049-54.

[14] Bornstein NM, Aronovich BD, Karepov VG, et al. The Tel Aviv stroke registry: 3600 consecutive cases. Stroke 1996;27(10):1770-3.

[15] Rastenyte D, Tuomilehto J, Domarkiene S, et al. Risk factors for death from stroke in middle-aged Lithuanian men: results from a 20 year prospective study. Stroke 1996;27(4):672-6.

[16] Sandercock PA, Warlow CP, Jones LN, et al. Predisposing factors for cerebral infarction: the Oxfordshire community stroke project. BMJ 1989;298:75-80.

[17] Shaper AG, Phillips AN, Pocock SJ, et al. Risk factors for stroke in middle aged British men. BMJ 1991;302:1111-5.

[18] Sridharan R. Risk factors for ischemic stroke: a case control analysis. Neuroepidemiology 1992;11(1): 24-30.

[19] Haheim LL, Holme I, Hjermann I, et al. Risk factors of stroke incidence and mortality. A 12 year follow-up of the Oslo study. Stroke 1993;24:1484-9.

[20] Burchfiel CM, Curb JD, Rodriguez BL, et al. Glucose intolerance and 22 year stroke incidence. The Honolulu heart program. Stroke 1994;25(5):951-7. 
[21] D’Agostino RB, Wolf PA, Belanger AJ, et al. Stroke risk profile: adjustment for antihypertensive medication. The Framingham study. Stroke 1994;25(1):40-3.

[22] Jamrozik K, Broadhurst RJ, Anderson CS, et al. The role of lifestyle factors in the etiology of stroke. A population-based case-control study in Perth, Western Australia. Stroke 1994;25(1):51-9.
[23] Wannamethee SG, Shapes AG. Patterns of alcohol intake and risk of stroke in middle aged British men. Stroke 1996;27(6):1033-9.

[24] Toole JF, Malinow MR, Chambless LE, et al. Lowering homocysteine in patients with ischemic stroke to prevent recurrent stroke, myocardial infarction, and death: the vitamin intervention for stroke prevention (VISP) randomized controlled trial. JAMA 2004;291(5):565-75. 